\title{
Frequency and latency measures of the generalization of punishment'
}

\author{
Eugene Winograd, Perrin S. Cohen and Brett K. Cole \\ COLUMBIA UNIVERSITY
}

\begin{abstract}
Abstraet
Punishment of rats was programmed to one of five click stimuli varying in repetition rate while a VI schedule was in effect to all stimuli. Gradients of median response latency, failures to respond, overall rate, and corrected rate with latency subtracted were compared for the last nine sessions. The different measures were highly correlated.
\end{abstract}

\section{Problem}

Blough (1963) has asked the question: what contributes to the rate differences that are seen in the typical generalization gradient obtained in extinction (Guttman \& Kalish, 1956)? He suggested that differential stimulus control is exerted only over those responses following long interresponse times (IRTs). An IRT analysis such as Blough's starts with the first response to a given stimulus, thereby eliminating any contribution made by latency to the shape of the generalization gradient. The present paper analyzes gradients of frequency and latency derived from punishment in a maintained generalization testing procedure (Winograd, 1965)。 Method

Five naive male albino rats approximately 120 days old at the start of the experiment were maintained at $80 \%$ of their free-feeding weights. The apparatus consisted of an operant conditioning chamber containing a lever and dipper mounted on one wall. Seven large floor grids together with the stainless steel walls of the chamber served as shock electrodes. The shock source was a Grason-Stadler Model 1064 shock generator and scrambler. The apparatus and general procedure have been described elsewhere (Winograd, 1965).

During a $100 \mathrm{~min}$. daily session, each of five click stimuli (S1: .62 pps; S2: 1.7 pps; S3: 4.8 pps; S4: 10.9 pps; S5: 26.7 pps) was presented eight times for a period of $2.5 \mathrm{~min}$. in a random sequence with no interval between stimulus changes. Data from the first presentation of each stimulus were discarded. Initially, the animals were given from 9-12 days of training on a variable-interval schedule of reinforcement with a mean of $1 \mathrm{~min}$. (VI 1) for a reinforcement of .02 cc of condensed milk. After all animals were responding at a reasonably constant rate in all stimuli, the punishment contingency was introduced. All responses made in S1 produced a $.25 \mathrm{ma}$ shock of $.5 \mathrm{sec}$. duration. The VI 1 schedule of milk reinforcement remained in effect at all times. Following 24-27 sessions at .25 ma, the shock intensity was increased to $.40 \mathrm{ma}$ for $39-42$ sessions. Two of the original five rats were discarded during the punishment sessions for failing to respond over many sessions.

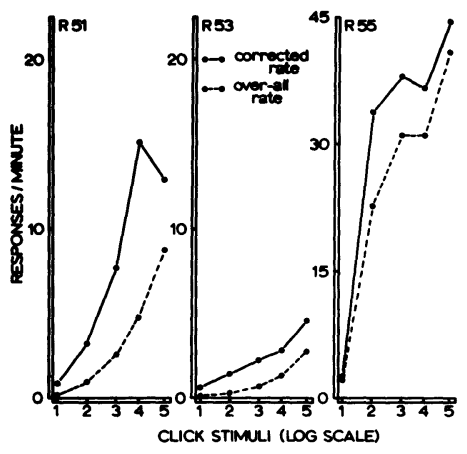

Fig. 1. Overall rate and correct rate of response as a function of click rate for three rats. Click values are given in the text.

\section{Results}

All the data to be presented are based on the last nine days of the punishment procedure at $.40 \mathrm{ma}$. There was little day to day variability at this stage in the experiment. Gradients based on rate of response are shown in Fig. 1 for three Ss: R 51, R 53, and R 55. The broken lines are based on the overall rate of response whereas the unbroken lines represent the corrected rate, computed as the rate of response after latency of the first response to each stimulus presentation was subtracted. Stimulus presentations to which no response occurred did not enter into the calculation of corrected rate. Figure 1 shows that the general shape of the gradients is independent of the method of computing rate, although reversals occur in the corrected rate gradients for $\mathbf{R} 51$ and $\mathrm{R}$ 55. If the frequency gradients are solely artifacts of latency differences, the corrected rate functions should be flat, which is clearly not the case.

The latency data are shown in Fig. 2, with the unbroken lines representing the median latency for 63 stimulus presentations for each $\mathrm{S}$ over the last nine sessions. The step-like functions for $\mathrm{R} 51$ and $\mathrm{R} 53$ reflect the fact that on the majority of trials these Ss did not respond at all within the 150-sec. presentations of the

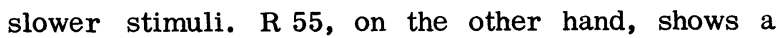
shorter latency to all stimuli with evidence of a steep gradient with an unaccounted for drop at $\mathrm{S} 1$. The rate of response for this $S$ can be seen in Fig. 1 to be considerably greater than the rate of the other Ss. In view of the large number of instances of failures to respond which were assigned latency values of $150 \mathrm{sec}$., the total number of failures to respond out of the 63 stimulus presentations was plotted for each stimulus and is shown as the broken lines in Fig. 2. This measure proves more sensitive to stimulus change than does 


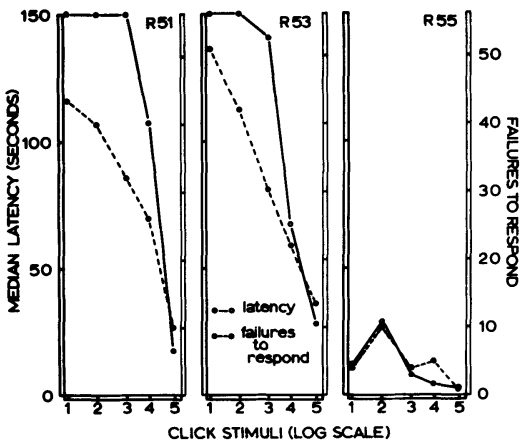

Fig. 2. Median latency and failures to respond within $150 \mathrm{sec}$. as a function of click rate for three rats. Click values are given in the text.

median latency for $\mathrm{R} 51$ and $\mathrm{R} 53$, and is highly correlated with median latency for $\mathrm{R} 55$.

In a study in which generalization gradients from pigeons to wavelength stimuli were studied, Hiss \& Thomas (1963) report that the median latency for the first five trials to each stimulus on which responding occurred during testing did not yield a significant gradient. Although the data are not presented here, the median latencies for on $1 \mathrm{y}$ those trials on which $\mathrm{S}$ did respond within $150 \mathrm{sec}$. did not yield orderly gradients.

\section{Diseussiom}

The present data indicate that generalization gradients based on frequency maintain their essential features when the contribution of latency is removed. Whether this generalization hold for other testing procedures is, of course, an empirical question. The data on latency suggest that for discrete-trial situations, in which latency is of necessity a major dependent variable, the seemingly gross measure of failures to respond within the allotted time may offer a more refined measure of generalization than does a central tendency measure of latency. This observation is particularly pertinent to situations in which variables having a decremental effect upon response strength are being investigated and latencies as long as the experimenter's observation interval are likely to occur.

\section{References}

BLOUGH, D. S. Interresponse time as a function of continuous variables: a new method and some data.J.exp. Anal. Behav., 1963, 6, 237-246.

GUTTMAN, N., \& KALISH, H. Discriminability and stimulus generalization. J. exp. Psychol., 1956, 51, 79-88.

HISS, R. H., \& THOMAS, D.R. Stimulus generalization as a function of testing procedure and response measure. J. exp. Psychol., 1963, $65,587-592$.

WINOGRAD, E. Maintained generalization testing of conditioned suppression. J. exp. Anal. Behav., 1965, 8, 47-51.

\section{Note}

1. This investigation was supported in part by Public Health Service Research Grant HD00933-03 from the National Institute of Mental Health.

\section{Editorial Note}

Because of the current and projected rate of publication of articles in the journal, Volume 2 will be brought to a close with Number 12 to be published on June 15. A Table of Contents will be included in that issue. The issue to appear on July 1 will be Number 1 of Volume 3 .

All individual and institutional subscribers who have entered their subscriptions for 1965 will receive Volume 3 without any additional charge. Subscriptions entered after July 1, however, will be charged at the rate of $\$ 10$ a volume for institutions and $\$ 5$ a volume for individuals. At that time, Volumes 1 and 2 become "backstock." Volume 1 will be priced at $\$ 10$ to all purchasers, and Volume 2 will be available at the regular volume price of $\$ 10$ for institutions and $\$ 5$ for individuals.

Further revisions in the subscription and volume structure will be required for 1966. It is planned to offer sectional subscriptions for alternate issues of the journal and to offer a reprint service for certain groups of articles. Details will be announced at a later date. 